\title{
MyD88 TIR domain signalosome interactions revealed by MicroED
}

\author{
M Clabbers ${ }^{1}$ \\ ${ }^{1}$ Stockholm University, Djursholm \\ clabbersm@gmail.com
}

Microcrystal electron diffraction (MicroED) has recently shown potential for structural biology, enabling structure determination from submicron-sized crystals of macromolecules and naturally self-assembled filaments of short peptide fragments, revealing the underlying mechanisms of interactions within assemblies present in cells. Here, we present the MicroED structure of the MyD88 TIR domain signalosome. Most Toll-like receptors (TLRs) and /interleukin-1 receptors (IL-1Rs) signal through the adaptors MAL and MyD88 to activate pro-inflammatory cytokines. It was previously observed that MAL induced the formation of thin crystalline arrays of MyD88TIR protofilaments with a diameter of typically $100 \mathrm{~nm}$. These microcrystals were too small for conventional x-ray crystallography, yet are highly suitable for structure elucidation by electron diffraction. We used MicroED to solve the structure via molecular replacement, based on a distantly related TIR domain homologue with only $30 \%$ sequence identity. The electrostatic potential map at $3.0 \AA$ resolution was of sufficient quality for accurate interpretation and rebuilding of the structural model. Several loop regions were remodelled and show conformations distinctly different from crystal structures of monomeric MyD88TIR. In parallel, the microcrystals were also characterized by serial X-ray free electron laser (XFEL) crystallography (2.3 $\AA$ resolution), confirming the interpretation of the model. Our results provide novel insights into TIR domain binding interactions that form the structural basis for signaling in the TLR and interleukin-1 receptor pathways. We anticipate MicroED to complement and extend existing methods in structural biology for a variety of crystalline samples that are too small for x-ray and neutron diffraction.

Acta Cryst. (2020). A76, a86 